\title{
Are theoretically calculated periods of vibration for skeletal structures error-free?
}

\author{
Sameh S.F. Mehanny* \\ Structural Engineering Department, Cairo University, \\ Dar Al-Handasah (Shair and Partners), Cairo, Egypt
}

(Received June 22, 2010, Revised July 25, 2011, Accepted July 27, 2011)

\begin{abstract}
Simplified equations for fundamental period of vibration of skeletal structures provided by most seismic design provisions suffer from the absence of any associated confidence levels and of any reference to their empirical basis. Therefore, such equations may typically give a sector of designers the false impression of yielding a fairly accurate value of the period of vibration. This paper, although not addressing simplified codes equations, introduces a set of mathematical equations utilizing the theory of error propagation and First-Order Second-Moment (FOSM) techniques to determine bounds on the relative error in theoretically calculated fundamental period of vibration of skeletal structures. In a complementary step, and for verification purposes, Monte Carlo simulation technique has been also applied. The latter, despite involving larger computational effort, is expected to provide more precise estimates than FOSM methods. Studies of parametric uncertainties applied to reinforced concrete frame bents - potentially idealized as SDOF systems - are conducted demonstrating the effect of randomness and uncertainty of various relevant properties, shaping both mass and stiffness, on the variance (i.e. relative error) in the estimated period of vibration. Correlation between mass and stiffness parameters - regarded as random variables - is also thoroughly discussed. According to achieved results, a relative error in the period of vibration in the order of $19 \%$ for new designs/constructions and of about $25 \%$ for existing structures for assessment purposes - and even climbing up to about $36 \%$ in some special applications and/or circumstances - is acknowledged when adopting estimates gathered from the literature for relative errors in the relevant random input variables.
\end{abstract}

Keywords: period of vibration; mass; stiffness; error propagation; monte carlo simulation

\section{Introduction}

The fundamental period of vibration for different civil structures (e.g. buildings, bridges, elevated tanks, etc...) represents the fingerprint for such structures identifying their dynamic characteristics and controlling their performance and response. This period does not only play a major role in predicting the expected behavior of these structures under any type of dynamic excitations, but it has also been traditionally used to estimate the equivalent lateral seismic design force as per various international seismic design codes and recommendations.

Recent research in seismic design universally pushes towards estimating such periods using either sophisticated analytical accurate formulae (e.g. via the Rayleigh quotient), or, computer eigen

\footnotetext{
* Corresponding author, Professor, E-mail: sameh.mehanny@stanfordalumni.org
} 
analysis instead of available ad-hoc conservative empirical equations currently extensively used by most designers and yet still approved and provided by codes. In their way to promote drifting to such accurate calculation methods predicting more realistic values, new generations of seismic design provisions (e.g. IBC 2003, UBC 1997 and ASCE 7-05) - mainly in the US practice - allow, as a first and modest step, for some relaxation in the considerably low-valued (Goel and Chopra 1997) fundamental period of vibration calculated by currently available approximate equations showing remarkable differences between "code-estimated" and "measured" period values for actual structures (Kwon and Kim 2010, Masi and Vona 2010, Pinho and Crowley 2009). But these provisions still do not fully permit ignoring such approximate period values and liberally using the analytically computed ones. They instead enforce an upper bound to the proposed allowed relaxation in the approximate low-valued code period. This bound, beside catering for the stiffening effect of nonstructural or "secondary" elements, is to protect against any uncertainty in the stiffness or mass modeling that may result in a corresponding un-conservative error in the period of vibration (predicting longer periods and consequently lower design forces leading to unsafe structures under actual excitations). Such upper ceiling to the period value thus admits the fairly noticeable margin of uncertainty and variance expected in the period of vibration - even if this period is theoretically calculated - and the consequent impact of such uncertainty on the designed facilities. It is however worth mentioning that in European seismic design practice such as in CEN (2004), a period calculated from basics of mechanics is allowed without limiting its value relative to the empirical (usually conservative) value. This applies both for equivalent static analysis as well as for modal response spectrum (linear dynamic) analysis.

The theme of this paper, geared towards the field of seismic-resistant design, is to quantify the error in the calculated period of single-mode dominant structures (representing a considerable percentage of civil structures) due to the error propagated through variation and uncertainty in the values of both mass and stiffness parameters. The paper adopts a Second-Order approximation technique to determine the expected value (including the anticipated built-in error) of the fundamental period of vibration of a given structure calculated from first principles of structural mechanics. It further applies First-Order Second-Moment (FOSM) approach to calculate the variance (representing the error) in the estimated period of vibration given the inherent error or uncertainty in its constituents. The FOSM results are checked versus those of the more accurate, yet computationally demanding, Monte Carlo simulation. Application to simple skeletal reinforced concrete structures is presented to demonstrate the effect of the error propagation on the period of vibration - such an important design and behavioral structural property. The focus of the paper is on single mode-dominant structures (idealized as SDOF systems) for which the equivalent lateral static analysis recommended by most seismic design codes worldwide is appropriate and fairly efficient. Correlation between mass and stiffness is carefully discussed and its effect on the estimated error in the fundamental period of vibration is also investigated.

\section{Propagation of uncertainty}

Uncertainty in estimated various structural properties due to either lack of knowledge or approximate modeling procedure directly affects the accuracy of the calculated and/or actual structural response. The probabilistic response of various structural systems or the probabilistic nature of any of their characteristics is generally expressed in terms of uncertain parameters 
associated with relevant structural properties. The uncertainty in these parameters is customarily expressed in terms of the coefficient of variation, C.O.V. The latter, representing the relative error in a given random variable, is usually estimated through unbiased sampling from large populations of the parameters of interest and is usually available in the literature for some common applications. The influence of uncertainty in these parameters is propagated in accordance with the functional relationships that relate them to the structural response or to the structural property of interest. The propagation of uncertainty may be accomplished by employing one or a combination of the following approaches: Taylor series-based perturbation techniques, stochastic finite element methods, Monte Carlo simulation, and Second-Moment approaches (Kareem 1988). In the special application related to random natural periods of vibration (i.e. random eigenvalue problems for large MDOF systems), considerable effort has already been spent in the probabilistic mechanics literature to most accurately estimate means of these eigenvalues (i.e. periods of vibration). A short but valuable survey of the literature in this arena is outlined in Adhikari and Friswell (2007) where it may be noted that more precise solution schemes including Monte Carlo simulation techniques (Adhikari and Friswell 2007) and methods based on polynomial chaos expansions (Ghosh et al. 2005) are currently promoted for tackling this highly sophisticated random problem. Such approaches are preferred than perturbation methods if expected level of uncertainty is fairly large.

In this paper, Second-Moment approach (namely, FOSM technique), supplemented by Monte Carlo simulation technique for validation purposes, are used to trace the uncertainties and quantify the propagation of error when estimating the fundamental period of vibration for the single modedominant skeletal structures of interest in this research.

\subsection{Second-moment techniques}

The Second-Moment techniques have traditionally provided practical and efficient means of analyzing probabilistic engineering mechanics problems (e.g. Ang and Tang 1984, Benjamin and Cornell 1970). The attractiveness of these techniques rests on the limited statistical information needed to analyze a problem. For example, only the first two statistical moments of a random variable are sufficient for the analysis. The expression for studied response or quantity is expanded in terms of the Taylor series; only up to the first or second-order terms are retained. In this paper, only the first-order terms are retained; the approximation is thus referred to as the First-Order Second-Moment (FOSM) approach. The discarded terms are functions of the second and higher order derivatives of the performance function, the variances and shapes of the probability density functions of the input variables, and the correlations among input variables. This means that the method is exact for linear performance functions, and that for a given degree of curvature, i.e. for nonlinear functions, the method is more accurate for smaller values of the variances (i.e. uncertainties) and/or if the input parameters distribution is Guassian. However, the FOSM method has been satisfactorily used by Ibarra (2003) in estimating variances in collapse capacities of structures due to randomness in System Parameters (SP) yet with fairly large inherent uncertainty (SP coefficient of variation of about $60 \%$ ). Despite these limitations, the FOSM method (or Taylor series-based perturbation technique) is sometimes preferred because it requires fairly smaller computational effort relative to the other equivalent approaches and it does not necessitate commitment to a probabilistic distribution of the input random variables (i.e. system parameters).

Given a function $Y$ of a number of random variables $X_{i}$ taking the following form 


$$
Y=g\left(X_{1}, X_{2}, X_{3}, \ldots, X_{i}, \ldots, X_{N}\right)
$$

one could express, in a FOSM format, the coefficient of variation, $\Omega_{Y}$, of the function $Y$ which represents the uncertainty (or relative error) in the expected value of $Y$ by

$$
\Omega_{Y}=\frac{1}{\bar{Y}}\left(\sum_{i=1}^{N}\left(\left.\frac{\partial g}{\partial x_{i}}\right|_{\bar{x}_{i}}\right)^{2} \bar{x}_{i}^{2} \Omega_{x_{i}}^{2}+\sum \sum_{i \neq j} \rho_{i j}\left(\left.\frac{\partial g}{\partial x_{i}}\right|_{\bar{x}_{i}}\right)\left(\left.\frac{\partial g}{\partial x_{j}}\right|_{\bar{x}_{j}}\right) \bar{x}_{i} \bar{x}_{j} \Omega_{x_{i}} \Omega_{x_{j}}\right)^{1 / 2}
$$

in which $\rho_{i j}$ is the correlation coefficient between $x_{i}$ and $x_{j}, \bar{Y}$ is the mean value of $Y, \bar{x}_{i}$ is the mean value of $x_{i}, \Omega_{x_{j}}$ is the C.O.V. of variable $x_{j}$, and $\partial g /\left.\partial x_{i}\right|_{\bar{x}_{i}}$ is the derivative of $g($.$) evaluated at the$ mean value of $x_{i}$. Note that Eq. (2) above may be re-written to alternatively express the FOSM approximation of the variance, $\sigma_{Y}^{2}$, of $Y$ in terms of the variance, $\sigma_{x_{i}}^{2}$, of different random variables, $x_{i}$.

$$
\sigma_{Y}^{2}=\sum_{i=1}^{N}\left(\left.\frac{\partial g}{\partial x_{i}}\right|_{\bar{x}_{i}}\right)^{2} \sigma_{x_{i}}^{2}+\sum \sum_{i \neq j} \rho_{i j}\left(\left.\frac{\partial g}{\partial x_{i}}\right|_{\bar{x}_{i}}\right)\left(\left.\frac{\partial g}{\partial x_{j}}\right|_{\bar{x}_{j}}\right) \sigma_{x_{i}} \sigma_{x_{j}}
$$

To expand the benefit of our study of propagation of uncertainty, and within the same line shown above, the second-order approximation of the Expected value of $Y, E[Y]$ or $\bar{Y}$ as shown above in Eq. (2), could be given by

$$
E[Y]=g\left(\bar{x}_{1}, \bar{x}_{2}, \bar{x}_{3}, \ldots, \bar{x}_{N}\right)+\left.\frac{1}{2} \sum_{i=1}^{N} \frac{\partial^{2} g}{\partial x_{i}{ }^{2}}\right|_{\bar{x}_{i}} \sigma_{x_{i}}^{2}+\left.\sum_{i=1}^{N} \sum_{j>i}^{N} \rho_{i j} \frac{\partial^{2} g}{\partial x_{i} \partial x_{j}}\right|_{\bar{x}_{i}, \bar{x}_{j}} \sigma_{x_{i}} \sigma_{x_{j}}
$$

\subsection{Monte Carlo simulation techniques}

Monte Carlo simulation involves sampling randomly to realize a large number of events in order to get reasonable estimates of statistical measures for the response parameter or the performance function of interest. Conversely to the FOSM method, the nonlinearity of the performance function of interest is not an issue - regarding the accuracy of results - in Monte Carlo (MC) simulation technique. However, the results of MC simulations are only accurate to the extent that the assumed Probability Distribution Functions (PDF) of considered random input variables are accurate. Moreover, the MC technique is computationally more demanding. Nevertheless, since the FOSM method introduced above is an approximation that simplifies the nature of the problem and that only satisfactorily works under a set of limitations, the validity of obtained results based on FOSM techniques needs to be checked through a more robust approach such as the Monte Carlo simulation.

\section{Application of the theory of error propagation to period calculation}

It is fairly obvious that the variation in mass, $M$, and stiffness, $K$, of a given structural system, is 
the source of error in fundamental period of vibration. Local errors in various individual random properties/variables shaping both mass and stiffness are already incorporated in global errors assumed (or estimated) for the mass and stiffness quantities regarded themselves as random variables.

\subsection{Formulation of the FOSM method}

In this section, the theory of error propagation in the context of the FOSM approach described above is implemented to derive the expected relative error in the final value of the period of vibration based on the errors incorporated in its constituents, namely: $M$ and $K$.

Applying the technique introduced in the previous section through Eqs. (1) to (4), and knowing that the theoretical value of the period of vibration of any single mode-dominant structural system (or of an idealized single degree of freedom SDOF oscillator) is simply given by the function

$$
T=2 \pi \sqrt{M / K}
$$

where $M$ is the total mass of the system (or simply the mass of the SDOF oscillator) and $K$ is the equivalent stiffness of this idealized SDOF oscillator, the second-order approximation of the expected value of $T$ may be estimated by manipulating

$$
E[T]=2 \pi \sqrt{\frac{\overline{\bar{M}}}{\bar{K}}}+\frac{1}{2}\left(\left.\frac{\partial^{2} T}{\partial M^{2}}\right|_{\bar{M}} \sigma_{M}^{2}+\left.\frac{\partial^{2} T}{\partial K^{2}}\right|_{\bar{K}} \sigma_{K}^{2}\right)+\left.\frac{\partial^{2} T}{\partial M \partial K}\right|_{\bar{M}, \bar{K}} \rho_{M, K} \sigma_{M} \sigma_{K}
$$

which results in the following simplified expression

$$
E[T]=\left(1-\frac{\Omega_{M}^{2}}{8}+\frac{3 \Omega_{K}^{2}}{8}-\frac{\rho_{M, K} \Omega_{M} \Omega_{K}}{4}\right) 2 \pi \sqrt{\frac{\bar{M}}{\bar{K}}}
$$

where $\sigma_{M}=\bar{M} \Omega_{M}$ and $\sigma_{K}=\bar{K} \Omega_{K} . \rho_{M, K}$ is the correlation coefficient between $M$ and $K ; \rho$ takes a value of 1 for fully (i.e. perfectly) correlated $M$ and $K$ and a value of "zero" for un-correlated mass and stiffness.

Similarly, by applying Eq. 3, the FOSM approximation of the variance of $T$ is

$$
\sigma_{T}^{2}=\left(\left.\frac{\partial T}{\partial M}\right|_{\bar{M}}\right)^{2} \sigma_{M}^{2}+\left(\left.\frac{\partial T}{\partial K}\right|_{\bar{K}}\right)^{2} \sigma_{K}^{2}+2\left(\left.\frac{\partial T}{\partial M}\right|_{\bar{M}}\right)\left(\left.\frac{\partial T}{\partial K}\right|_{\bar{K}}\right) \rho_{M, K} \sigma_{M} \sigma_{K}
$$

The first two terms represent the contribution of the variance in $M$ and $K$ to the variance in $T$. The last term represents, however, the contribution of the correlation between $M$ and $K$ to the variance of the period $T$.

Finally, combining Eq. (7) and the solution of Eq. (8), the C.O.V., $\Omega_{T}$, of the period $T$ (representing the relative error in the expected mean value of $T$ ) may be given by

$$
\Omega_{T}=\sqrt{\Omega_{M}^{2}+\Omega_{K}^{2}-2 \rho_{M, K} \Omega_{M} \Omega_{K}} /\left(2-\frac{\Omega_{M}^{2}}{4}+\frac{3 \Omega_{K}^{2}}{4}-\frac{\rho_{M, K} \Omega_{M} \Omega_{K}}{2}\right)
$$




\subsection{Implementation of Monte Carlo technique}

In the present research, to derive the expected relative error in the value of the period of vibration based on the errors incorporated in its constituents through MC simulation technique, the random input variables $(M$ and $K$ ) assume 10,000 different randomly generated values following selected appropriate PDFs with reasonably assumed statistical properties (e.g. mean and C.O.V., i.e. $\Omega$ ) gathered from the literature as will be discussed in details in a following section. MATLAB is used as a computational platform to generate relevant random input and to calculate variations, $\Omega_{T}$, in the resulting period of vibration. Random generation of each of the input variables $(M$ and $K)$ is first independently performed to model un-correlated scenario for both $M$ and $K$. Then, to realize the perfectly correlated situation, an uncertain term, $\varepsilon$, with mean value, $E[\varepsilon]=1.0$ and a given $\Omega_{\varepsilon}$ applicable to both $M$ and $K$ is first randomly generated adopting the appropriate PDF. In a following step, the actual mean value of $M$ and $K$ is multiplied by the randomly generated uncertainty term, $\varepsilon$, thus capturing perfect correlation between $M$ and $K$.

\section{Correlation between mass and stiffness}

The fundamental period of vibration of a given structure approximated (i.e. idealized) as a Single Degree of Freedom SDOF system is a function of its vibrating lumped mass and the lateral stiffness of its supporting system as shown in Eq. (5). The problem of statistical correlation between mass and stiffness of such structures is relatively debatable (Deierlein and Cornell, personal communication, August, 2006). In a design sense, as will be described in the sequel, one could argue that there is some statistical correlation between the two structural properties. However, if one were looking at the randomness in properties, the correlation would be rather small and may be assumed marginal or even negligible.

Correlation in a design sense: Since presumably the stiffness of a system is correlated with its strength, and since for seismic design purposes the required strength is a function of supported mass, then from a design standpoint, one could presume some correlation between the two variables in a general sense. Therefore, for building systems of a certain type, e.g. moment frames, or for bridges with pier-to-girder monolithic connections, one would expect some correlation between mass and stiffness in a design context.

No-correlation in randomness for an existing system: On the other hand, once a system is designed and the nominal mass and stiffness are fixed, further variations in the two would be uncorrelated. For instance, since most of the mass is due to floor framing in buildings and decking system for bridges and superimposed dead load, one would not expect that random increases in these values would be associated with significant changes in stiffness. Similarly, many of the major variations in stiffness (e.g. assumption on effective stiffness, EI, foundation rigidity, connection rigidity, composite beam action, etc...) would not be associated with a random change in mass. One could argue though that member sizes that are selected larger than nominal during design process (e.g. slightly larger columns or walls) would increase both stiffness and mass, however, percentagewise their impact on mass would be minimal.

To further elaborate, one should realize from the formal statistical definition of the term "correlation" that it is related to the expected value of random deviations from the means of the mutually correlated variables. One could still argue that this is not the same as saying, for example, 
if in a given design the specified slab thickness is doubled, then the frame will also get stiffer because there is a need to increase columns dimensions to carry the heavier mass and its associated inertial effect. This latter situation would rather represent a "change in the means" of mass and stiffness and not "deviations from the mean" as implied by the "correlation" statistical concept. In a practical sense, having perfect correlation between two random variables implies that if one variable deviates from its mean by a standard deviation, $\sigma$, then so will the other. However, each variable still has its own marginal distribution (e.g. mean and $\sigma$ ), but the two variables also display this close coupling of their individual deviations relative to the mean irrespective of any changes in their individual "means".

For the purpose of this paper, both "perfectly correlated" and "un-correlated" mass and stiffness parameters will be investigated in order to quantify the effect of such correlation, if any, on the severity of the error propagating and accordingly impairing the accuracy of the period of vibration. "Perfectly correlated" and "un-correlated" situations represent, in a sense, lower and upper bounds, respectively, to the error (i.e. the variance) in the estimated period of vibration.

\section{Example of simple skeletal structures likely approximated by SDOF systems}

The error propagation analysis described in Sections 2 and 3 above is further applied herein for demonstration purposes to single and/or multi-bay reinforced concrete frame bents. Investigated frames are selected as single-story frames with high potential to be idealized as SDOF systems. By selecting such simple structures one could avoid unnecessary complications that may conceal the main idea of studying and demonstrating the propagation of parametric uncertainties while determining the period of vibration of skeletal structural systems, for seismic design purposes, using either the introduced FOSM approach or MC simulation technique. Such case study frames are commonly encountered in bridges, warehouses, industrial facilities, single-story dwellings, etc... which constitute a considerable percentage of civil structures.

The stiffness parameter, $K$, is theoretically derived for this specific case using elementary structural mechanics and may be given as

$$
K=\sum_{c} \alpha \frac{E_{c} I_{c}}{H^{3}}
$$

Summation is over all columns of the frame. $\alpha$ is a problem-dependent constant coefficient which is a function of end conditions of the columns; these end conditions are implicitly depending on the beam-to-column stiffness ratio. $\alpha$ is considered as a deterministic constant in the present research. $I_{c}$ is the in-plane moment of inertia for each of the columns, and $H$ is the column height from top of foundation to beam level. For the purpose of this research, a simple idealized example - easy to interpret - of a single-bay one-story reinforced concrete frame is selected. Assuming both columns with same height and same cross section and assuming the beam infinitely rigid with respect to the columns and that the columns are fixed to the foundation, the summation symbol, $\Sigma$, in Eq. (10) is dropped out and the coefficient a takes the deterministic value of 24. However, the symbol $\alpha$ will be kept in the sequel without numerical substitution for generality.

$E_{c}$ is the modulus of elasticity for concrete. It is generally given by different design codes worldwide (e.g. ACI 318 2005) as a function of the square root of the compressive strength, $f_{c u}$, of 
concrete and may be written as

$$
E_{c}=\beta \sqrt{f_{c u}}
$$

where $\beta$ is a constant that varies from code to code partly depending on the quality of concrete industry in the local construction practice.

Applying Eq. (4), the second-order approximation of the expected value of $K$ may be estimated by solving

$$
\begin{aligned}
E[K]= & \frac{\alpha \beta \sqrt{\bar{f}_{c u}} \bar{I}_{c}}{\bar{H}^{3}}+\frac{1}{2}\left(\left.\frac{\partial^{2} K}{\partial f_{c u}{ }^{2}}\right|_{\bar{f}_{c u}} \sigma_{f_{c u}}^{2}+\left.\frac{\partial^{2} K}{\partial I_{c}^{2}}\right|_{\bar{I}_{c}} \sigma_{I_{c}}^{2}+\left.\frac{\partial^{2} K}{\partial H^{2}}\right|_{\bar{H}} \sigma_{H}^{2}\right) \\
& +\left.\frac{\partial^{2} K}{\partial f_{c u} \partial I_{c}}\right|_{\bar{f}_{c u}, \bar{I}_{c}} \rho_{f_{c u}, I_{c}} \sigma_{f_{c u}} \sigma_{I_{c}}+\left.\frac{\partial^{2} K}{\partial f_{c u} \partial H}\right|_{\bar{f}_{c u}, \bar{H}} \rho_{f_{c u}, H} \sigma_{f_{c u}} \sigma_{H} \\
& +\left.\frac{\partial^{2} K}{\partial H \partial I_{c}}\right|_{\bar{H}_{,} \bar{I}_{c}} \rho_{H, I_{c}} \sigma_{H} \sigma_{I_{c}}
\end{aligned}
$$

which may be simplified as

$$
E[K]=\left(1-\frac{\Omega_{f_{c u}}^{2}}{8}+6 \Omega_{H}^{2}+\frac{\rho_{f_{c u}, I_{c}} \Omega_{f_{c u}} \Omega_{I_{c}}}{2}-\frac{3 \rho_{f_{c u}, H} \Omega_{f_{c u}} \Omega_{H}}{2}-3 \rho_{H, I_{c}} \Omega_{H} \Omega_{I_{c}}\right) \frac{\alpha \beta \sqrt{\bar{f}_{c u}} \bar{I}_{c}}{\bar{H}^{3}}
$$

with same system of notations previously defined. Note that, for instance, if $H$ is a deterministic constant, i.e. error free, instead of the general assumption of being a random variable, the coefficient of variation $\Omega_{H}$ vanishes and Eq. (13) may be reduced to

$$
E[K]=\left(1-\frac{\Omega_{f_{c u}}^{2}}{8}+\frac{\rho_{f_{c u}, I_{c}} \Omega_{f_{c u}} \Omega_{I_{c}}}{2}\right) \frac{\alpha \beta \sqrt{\bar{f}_{c u}} \bar{I}_{c}}{\bar{H}^{3}}
$$

Similarly, by applying Eq. (3), the FOSM approximation of the variance of $K$ expressed in terms of Eqs. (10) and (11) is

$$
\begin{aligned}
\sigma_{K}^{2} & =\left(\left.\frac{\partial K}{\partial f_{c u}}\right|_{\bar{f}_{c u}}\right)^{2} \sigma_{f_{c u}}^{2}+\left(\left.\frac{\partial K}{\partial I_{c}}\right|_{\bar{I}_{c}}\right)^{2} \sigma_{I_{c}}^{2}+\left(\left.\frac{\partial K}{\partial H}\right|_{\bar{H}}\right)^{2} \sigma_{H}^{2} \\
& +2\left(\left.\frac{\partial K}{\partial f_{c u}}\right|_{\bar{f}_{c u}}\right)\left(\left.\frac{\partial K}{\partial I_{c}}\right|_{\bar{I}_{c}}\right) \rho_{f_{c u}, I_{c}} \sigma_{f_{c u}} \sigma_{I_{c}}+2\left(\left.\frac{\partial K}{\partial f_{c u}}\right|_{\bar{f}_{c u}}\right)\left(\left.\frac{\partial K}{\partial H}\right|_{\bar{H}}\right) \rho_{f_{c u}, H} \sigma_{f_{c u}} \sigma_{H} \\
& +2\left(\left.\frac{\partial K}{\partial H}\right|_{\bar{H}}\right)\left(\left.\frac{\partial K}{\partial I_{c}}\right|_{\bar{I}_{c}}\right) \rho_{H, I_{c}} \sigma_{H} \sigma_{I_{c}}
\end{aligned}
$$


Similarly to what has been performed to get $\Omega_{T}$, solving Eq. (15) and making use of the result given by Eq. (13) or (14), the C.O.V. of the stiffness random parameter may be given as follows

$$
\Omega_{K}=\sqrt{\frac{\Omega_{f_{c u}}^{2}}{4}+\Omega_{I_{c}}^{2}+\rho_{f_{c u}, I_{c}} \Omega_{f_{c u}} \Omega_{I_{c}}} /\left(1-\frac{\Omega_{f_{c u}}^{2}}{8}+\frac{\rho_{f_{c u}, I_{c}} \Omega_{f_{c u}} \Omega_{I_{c}}}{2}\right)
$$

Note that Eq. (16) above assumes again a deterministic value of the variable $H$, i.e. ignoring any uncertainty or relative error in the columns height for the subject frame $\left(\Omega_{H}=0\right)$. Such error-free assumption for $H$ is just for the sake of presenting the resulting variation in the stiffness parameter in a concise format. However, in the numerical analysis section, results addressing $H$ as a random variable are also presented and evaluated.

To conclude, one could say that to determine the relative error in the expected value of the period of vibration $T$, Eq. (9) may be directly used if an estimation of $\Omega_{K}$ is adequately available to the user. Otherwise, $\Omega_{K}$ could be first estimated through applying Eq. (16) based on reasonable estimates - fairly available in the literature - of C.O.V. of relevant random variables, $f_{c u}$ and $I_{c}$. Then, as a complimentary step, the user could plug in Eq. (9) to determine a final estimate for $\Omega_{T}$.

Again, a MC simulation technique is carried out to randomly generate a wealth of values of the stiffness $K$ relying on adequate PDFs of relevant input random variables: $f_{c u}, I_{c}$ and $H$. Estimates of the variation in the resulting $K$ values due to uncertainty in the input parameters are then recovered.

\section{Assessment of coefficient of variation for various considered random parameters}

Estimates for C.O.V., i.e. $\Omega$ and adequate Probability Distribution Functions (PDF) for various considered random variables benchmarking the relative error (or uncertainty) in theoretically calculated fundamental period of vibration for reinforced concrete skeletal structures are compiled from the literature.

First, the structure mass is considered an uncertain (i.e. random) variable for the following reasons: as-built members dimensions may vary from those shown on the design drawings, unit weights of construction materials are imperfectly known; location and dimension of non-structural elements are generally uncertain, and actual structure components may vary from those assumed in the design, e.g. layers of roofing are often added during the life of a building which can significantly affect dead load. Quantification of uncertainty in the structure mass is an integration of uncertainties in the above-mentioned factors. However, for simplicity, most researchers in the literature considered mass uncertainty as a whole, as per Ellingwood and his co-workers (Ellingwood et al. 1980), following a representative PDF (usually Gaussian) with an assumed typical C.O.V. of 0.10 applied to the mean value of the nominal calculated mass, $M$ (e.g. Haselton and Deierlein 2005, Shaikhutdinov 2004). To avoid unrealistic negative values for such positive structural parameter, $M$, that may inadvertently assume spurious non-vanishing probabilities, a Lognormal PDF is therefore instead adopted herein for the random mass parameter (Soize 2000, Tarantola 2005).

Structure stiffness is comparably an uncertain variable for reasons similar to those listed above for mass: as-built dimensions, contribution of non-structural elements, properties of construction materials, beside other behavioral factors due to level of concrete cracking, effective inertia, etc... 
Different researchers suggest a range for the variation in $K$. For example, a value of $\Omega_{K}=0.27$ is adopted by Kareem (1980) for reinforced concrete tall chimneys with a circular cross-section. On the other hand, for regular reinforced concrete skeletal structures, other researchers suggest a value of $\Omega_{K}$ in the order of 0.36 (Haselton and Deierlein 2005, Fardis and Biskinis 2003, Panagiotakos and Fardis 2001) with $K$ following a Lognormal distribution. Note that this value is reported to apply for isolated members and is based on the member's initial stiffness. Such value is expected to increase after occurrence of some structural damage and non-linearity during the lifetime of the structure. To recapitulate, $\Omega_{K}$ of 0.36 may accordingly be adopted for a new design while an expected higher value may be assumed (or estimated) for an existing structure.

On the other hand, instead of adopting a lumped and directly assumed value for $\Omega_{K}$, an educated estimate may be derived for simple skeletal structures, potentially idealized as SDOF systems, through an integration of the variance in the various random components of the variable $K$. Such components - as introduced in section 5 and Eq. (10) - include moment of inertia, $I_{c}$, of the columns of the frame, its effective height, $H$, and a material property considered herein as the compressive strength of concrete, $f_{c u}$, for reinforced concrete structures.

Most researchers collectively agree upon a reasonable estimate for the variation in $f_{c u}$. Uncertainty in this random material property may be quantified by $\Omega_{f_{c u}}=0.18$ (Kareem 1988, Shaikhutdinov 2004). Such estimate is based on previous research by Mirza et al. (1979). For the same practical reasons mentioned above and in order to avoid non-vanishing probabilities for unrealistic negative values of this positive mechanical property, a Lognormal PDF is assumed for the random variable, $f_{c u}$ (Soize 2000 and Tarantola 2005).

Remaining random variables affecting the stiffness random parameter, $K$, are the geometrical properties of the case study frame: $I_{c}$ and $H$. Uncertainty in these variables may be derived from available literature (Kareem 1988, Haselton and Deierlein 2005). As a result, $I_{c}$ is assumed to follow a Lognormal distribution with $\Omega_{I_{c}}$ falling in a wide range between 0.16 (for the case of gross uncracked inertia) and 0.35 (accounting for effective inertia with cracking-induced uncertainty). $\Omega_{H}$ is in the order of 0.04 , where $H$, as well following a Lognormal PDF, is the column height measured for structural analysis purposes from top of foundation to beam bottom level. Such C.O.V. of $H$ may assume larger values to account for other additional implicit uncertainty related to modeling issues trying to capture the dynamic behavior of the frame. In other words, $\Omega_{H}$ may depend on whether the height considered extends to the bottom of beam or, instead, to the beam centerline; whether such modeling assumption depends on the beam-to-column stiffness ratio or not, etc

\section{Numerical analysis and discussions}

Effect of parametric uncertainties on the relative error of theoretically calculated fundamental period of vibration for skeletal structures is presented in this section. All possible permutations - in a fairly wide range - for the coefficients of variation $\Omega_{M}$ and $\Omega_{K}$ of random variables $M$ and $K$, respectively, are studied. As previously stated, $\Omega_{M}$ and $\Omega_{K}$ represent the relative error in the corresponding expected (mean) value of mass and stiffness random parameters, respectively. The investigated range for C.O.V. (i.e. $\Omega$ ) encompasses values between, and including, 0 (denoting an error-free variable) and 0.5 (representing a considerable relative error or variation). Such broad range, that may be overestimating the realistic variation in either $M$ or $K$, is however adopted to model practically all plausible bounds on the relative error, $\Omega_{T}$, in the resulting period of vibration, 
T. Specific values of C.O.V. for different considered random variables inferred from information available in the literature are also implemented herein to model discrete situations resulting in particular relative error in the period of vibration.

A set of three-dimensional surface plots is generated using MATLAB - applying the FOSM method introduced herein and considering various scenarios - for the relative error, $\Omega_{T}$, regarded as a continuous function of corresponding relative errors in $M$ and $K$ parameters. Different studied scenarios include "un-correlated" and "perfectly correlated" relevant random variables for the following two cases: (1) $\Omega_{M}$ and $\Omega_{K}$ directly assigned, as well as (2) $\Omega_{M}$ directly assigned while $\Omega_{K}$ computed from the corresponding C.O.V. of its basic components. Moreover, to ensure a precise quantification of $\Omega_{T}$, the more accurate Monte Carlo simulation technique has been applied to a few selected discrete variations of the relevant random input variables. Note that, although the statistical assumption of perfectly correlated $M$ and $K$ is highly unlikely as previously discussed, it is included herein to draw the full picture with lower and upper limits to the relative error, $\Omega_{T}$.

Figs. 1 and 2 show the continuous variation in the relative error, $\Omega_{T}$, due to mutual uncertainty in mass and stiffness parameters represented by $\Omega_{M}$ and $\Omega_{K}$ for the un-correlated and perfectly correlated assumptions, respectively, via the FOSM method. It may be noted that $\Omega_{T}$ for uncorrelated mass and stiffness is constantly larger than its value for the perfectly correlated assumption. This applies to all combinations of non-zero values of $\Omega_{M}$ and $\Omega_{K}$. It may be also observed from Fig. 2 that $\Omega_{T}$ always assumes a non-zero value for the scenario of perfectly correlated variables for the studied wide range of joint relative errors, $\Omega_{M}$ and $\Omega_{K}$, excluding the case where coincidentally $\Omega_{M}=\Omega_{K}$. This latter situation with equal relative error in both mass and stiffness parameters, along with the highly unlikely perfect positive correlation assumption between these two random variables, causes their relative errors to cancel out (refer to Eq. (9)). The same result has been equally observed when performing the MC simulation technique.

It is also worth mentioning that, when following FOSM methods, if either mass or stiffness is considered as a deterministic (i.e. error free) parameter for a special application, the resulting error, $\Omega_{T}$, in the period of vibration is about half the corresponding relative error in the other random variable (be it stiffness or mass). Referring to Eq. (5), the square root relationship is responsible for reducing (cutting to half) the resulting relative error propagated in the period $T$ with respect to associated built-in relative errors in $M$ and $K$. On the other hand, if applying the more accurate MC

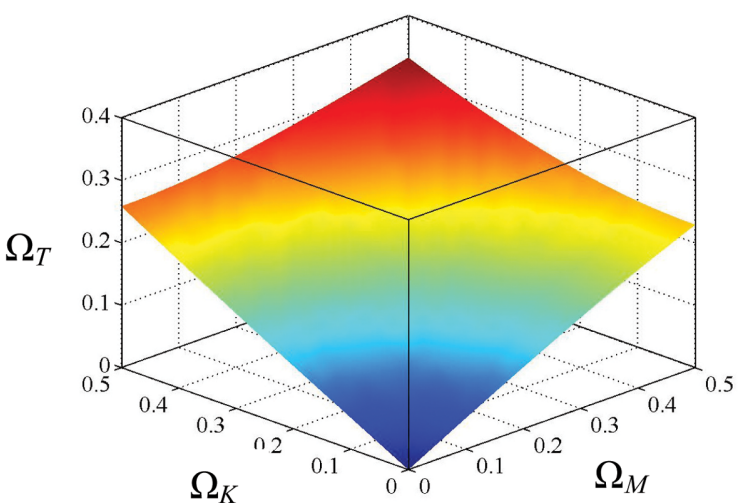

Fig. 1 Relative error in $T$ - Case of un-correlated mass and stiffness parameters

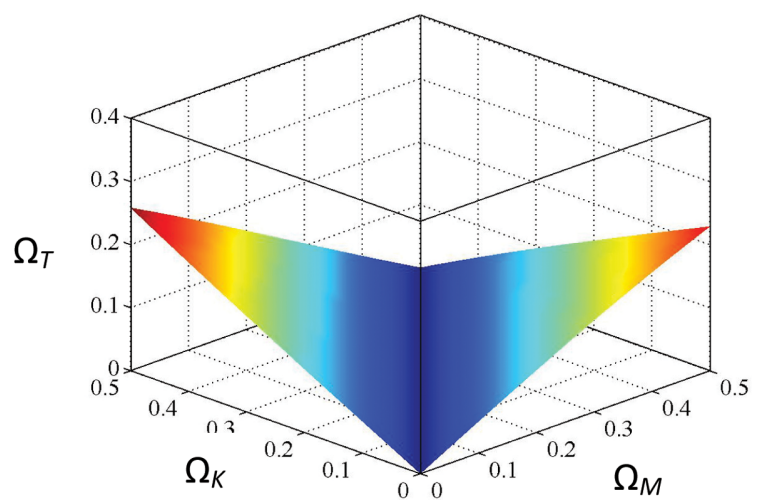

Fig. 2 Relative error in $T$ - Case of perfectly correlated mass and stiffness parameters 
simulation techniques that work better for nonlinear functions, it may be observed that the contribution of the relative error in either $M$ or $K$, independently considered, to the relative error in $T, \Omega_{T}$, is almost alike. In other words, as may be noted from Table $1, \Omega_{T}$ for random $M$ associated with a deterministic $K$ (or with a specific $\Omega_{K}$ ) is nearly identical to that for random $K$ along with a deterministic (i.e. error free) $M$ (or with a specific $\Omega_{M}$ ). This may be easily observed for instance if consulting the values of $\Omega_{T}$ in any row in Tables 1(a) through 1(d) showing the effect on the relative error in $T$ caused by different $\Omega_{K}$ values associated with a specific value of $\Omega_{M}$ (say 0.3 ) and comparing them to the corresponding values of $\Omega_{T}$ due to different values of $\Omega_{M}$ associated with the specific value of 0.3 for $\Omega_{K}$. The difference in these $\Omega_{T}$ values is practically negligible even at larger uncertainties considered herein (i.e. at $\Omega_{M}$ and $\Omega_{K}$ values as high as 0.5 ). As a general observation, for all typical studied variations encountered in the present research, both the FOSM method which is much less computationally demanding, and the $\mathrm{MC}$ technique give fairly comparable results.

Specific values of expected relative errors in $M$ and $K$ for reinforced concrete skeletal structures retrieved from available literature are particularly determined. For example, for the highly likely scenario of un-correlated $M$ and $K, \Omega_{T}$ assumes a value of 0.18 (Fig. 1) and 0.19 (as inferred from Table 1) when applying FOSM and MC techniques, respectively, for $\Omega_{M}=0.1$ and $\Omega_{K}=0.36$ representing new designs. On the other hand, for $\Omega_{K}=0.5$ accounting for probable additional variation in stiffness due to expected random global damage and nonlinearity all over the structure during its lifetime (i.e. case of assessment of existing structures), $\Omega_{T}$ scores an increase of about $33 \%\left(\Omega_{T}=0.24\right)$ when FOSM is used and almost practically same percentage increase of about $32 \%\left(\Omega_{T}=0.25\right)$ if MC simulation is applied as shown in Table 1. This outcome reinforces the conclusion that FOSM results - when this rather approximate but less computationally demanding perturbation technique is applied to the stochastic eigenvalue problem considered in this research are fairly accurate if compared to those of the more reliable MC simulation technique usually

Table 1(a) Values of $\Omega_{T} \times 100$ from MC simulation (un-correlated $M$ and $K$ ) $-1,000$ simulations

\begin{tabular}{c|ccccccc}
\hline \hline$\Omega_{M}$ & $\Omega_{K}$ & 0 & 0.1 & 0.2 & 0.3 & 0.4 & 0.5 \\
\hline 0 & 0.0 & 5.1 & 9.9 & 14.4 & 18.8 & 23.9 \\
0.1 & 5.0 & 7.3 & 11.1 & 15.3 & 19.6 & 24.4 \\
0.2 & 9.3 & 10.6 & 13.5 & 17.5 & 21.5 & 25.9 \\
0.3 & 15.2 & 16.2 & 17.5 & 21.1 & 24.1 & 28.4 \\
0.4 & 19.3 & 20.2 & 21.5 & 23.9 & 26.4 & 30.8 \\
0.5 & 23.2 & 23.6 & 25.8 & 28.2 & 30.0 & 33.3 \\
\hline
\end{tabular}

Table 1(b) Values of $\Omega_{T} \times 100$ from MC simulation (un-correlated $M$ and $K$ ) - 10,000 simulations

\begin{tabular}{c|ccccccc}
\hline \hline$\Omega_{M}$ & $\Omega_{K}$ & 0 & 0.1 & 0.2 & 0.3 & 0.4 & 0.5 \\
\hline 0 & 0.0 & 4.9 & 9.8 & 14.8 & 19.6 & 24.2 \\
0.1 & 5.0 & 7.0 & 11.0 & 15.6 & 20.3 & 24.8 \\
0.2 & 9.9 & 11.2 & 14.0 & 17.7 & 22.1 & 26.3 \\
0.3 & 14.9 & 15.9 & 18.0 & 21.1 & 24.9 & 28.7 \\
0.4 & 19.5 & 20.1 & 21.9 & 24.9 & 27.8 & 31.6 \\
0.5 & 24.1 & 24.7 & 26.1 & 28.5 & 31.2 & 34.6 \\
\hline
\end{tabular}


Table 1(c) Values of $\Omega_{T} \times 100$ from MC simulation (un-correlated $M$ and $K$ ) $-100,000$ simulations

\begin{tabular}{cccccccc}
\hline \hline$\Omega_{M}$ & $\Omega_{K}$ & 0 & 0.1 & 0.2 & 0.3 & 0.4 & 0.5 \\
\hline 0 & 0.0 & 5.0 & 9.9 & 14.8 & 19.5 & 24.0 \\
0.1 & 5.0 & 7.1 & 11.1 & 15.6 & 20.1 & 24.6 \\
0.2 & 9.9 & 11.1 & 14.1 & 17.9 & 22.0 & 26.1 \\
0.3 & 14.8 & 15.6 & 17.9 & 21.0 & 24.6 & 28.5 \\
0.4 & 19.4 & 20.0 & 21.8 & 24.6 & 27.7 & 31.3 \\
0.5 & 23.8 & 24.4 & 25.9 & 28.3 & 31.2 & 34.3 \\
\hline
\end{tabular}

Table 1(d) Values of $\Omega_{T} \times 100$ from MC simulation (un-correlated $M$ and $K$ ) $-250,000$ simulations

\begin{tabular}{c|ccccccc}
\hline \hline$\Omega_{M}$ & $\Omega_{K}$ & 0 & 0.1 & 0.2 & 0.3 & 0.4 & 0.5 \\
\hline 0 & 0.0 & 5.0 & 9.9 & 14.8 & 19.4 & 24.0 \\
0.1 & 5.0 & 7.1 & 11.1 & 15.6 & 20.0 & 24.5 \\
0.2 & 9.9 & 11.1 & 14.0 & 17.8 & 21.9 & 26.1 \\
0.3 & 14.8 & 15.6 & 17.8 & 20.9 & 24.5 & 28.4 \\
0.4 & 19.5 & 20.1 & 21.9 & 24.6 & 27.8 & 31.2 \\
0.5 & 23.9 & 24.4 & 26.0 & 28.4 & 31.1 & 34.3 \\
\hline
\end{tabular}

known for better accuracy at large uncertainties of the associated random variables and/or high intrinsic nonlinearity in the response function of interest.

The convergence of the $\mathrm{MC}$ simulation procedure performed herein with respect to the number of simulations has been also assessed through a systematic numerical sensitivity analysis. Four different numbers of realizations have been performed for the case of un-correlated random parameters, $M$ and $K$, namely: 1,000, 10,000, 100,000 and 250,000 simulations. Results of these simulations are shown in Tables 1(a) through 1(d), respectively. Resulting $\Omega_{T}$ values retrieved from the four cases are fairly identical with the differences between corresponding variations practically insignificant. This means that (1) MC simulation has converged to accurate and stable estimates of the C.O.V. of the randomly computed period of vibration, $T$, very comparable to those retrieved from applying the FOSM approach, and that (2) such resulting $\Omega_{T}$ values are not spuriously bounded as a result of halting the simulation after an arbitrary finite number of realizations.

In a complementary effort, the relative error in $K$ is computed by locally applying the FOSM technique introduced in Eqs. (1) to (3) and further manipulated for the case study example of a simple SDOF reinforced concrete skeletal frame (Eqs. (15) and (16)). Random variables of interest for this application are: the frame height $H$; the moment of inertia, $I_{c}$, of the columns of the frame; and the compressive strength of concrete, $f_{c u}$. Each variable shares differently in the value of $K$ (Eq. (10)) and hence in its associated relative error. For example, $H$ is raised to the power 3 with its relative error therefore contributing the most to $\Omega_{K} ; I_{c}$ is raised to the power 1 which lessens the negative impact of its uncertainty on the overall relative error in $K$; while, $f_{\text {cu }}$ shows up in the functional with a square root (i.e. raised to the power of $1 / 2$ ) thus contributing the least to $\Omega_{K}$.

Figs. 3 and 4 based on applying the FOSM method describe the continuous variation in the relative error, $\Omega_{K}$, due to joint uncertainty in $I_{c}$ and $f_{c u}$ introduced by $\Omega_{I_{c}}$ and $\Omega_{f_{c u}}$ for the uncorrelated and perfectly correlated hypotheses, respectively, for the case with a deterministic height $H$ of the subject frame. It may be easily observed that the assumption of perfect correlation between 


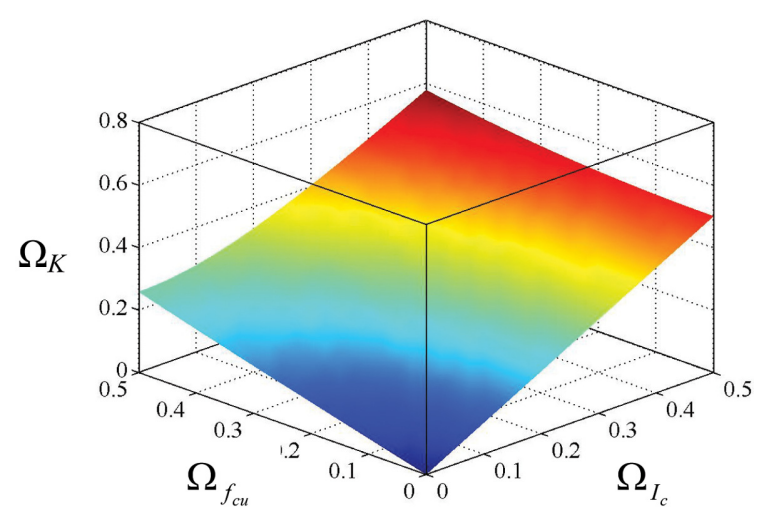

Fig. 3 Relative error in $K$ - Case of un-correlated $I_{c}$ and $f_{c u}$ with $\Omega_{H}=0$

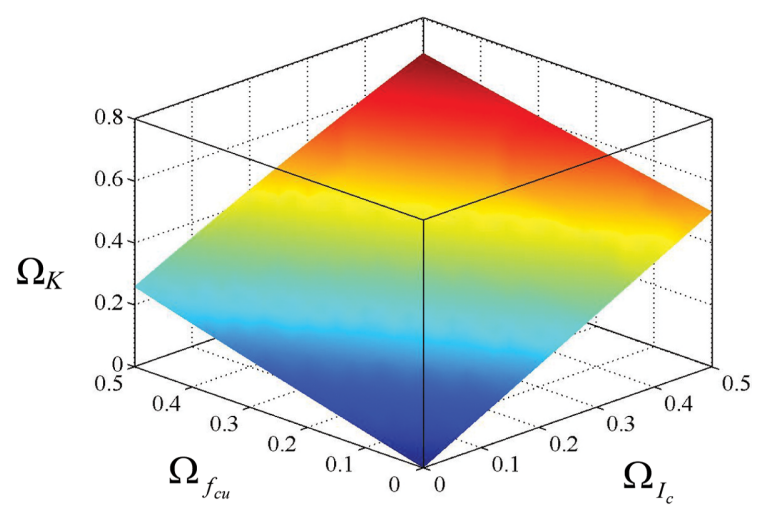

Fig. 4 Relative error in $K$ - Case of perfectly correlated $I_{c}$ and $f_{c u}$ with $\Omega_{H}=0$

Table 2 Values of $\Omega_{K} \times 100$ from MC simulation (un-correlated $f_{c u}$ and $I_{c}$ - deterministic $H$ )

\begin{tabular}{ccccccc}
\hline \hline$\Omega_{f_{\text {cu }}} \Omega_{I_{c}}$ & 0 & 0.1 & 0.2 & 0.3 & 0.4 & 0.5 \\
\hline 0 & 0.0 & 10.0 & 20.1 & 29.5 & 39.9 & 49.0 \\
0.1 & 5.0 & 11.2 & 20.8 & 30.0 & 40.2 & 49.3 \\
0.2 & 9.9 & 14.1 & 22.5 & 31.3 & 41.4 & 50.0 \\
0.3 & 14.9 & 18.1 & 24.9 & 33.4 & 42.6 & 51.9 \\
0.4 & 19.5 & 22.0 & 28.3 & 35.8 & 45.2 & 53.3 \\
0.5 & 24.2 & 26.3 & 31.7 & 38.8 & 47.4 & 55.3 \\
\hline
\end{tabular}

Table 3 Values of $\Omega_{K} \times 100$ from MC simulation (perfectly correlated $f_{c u}$ and $I_{c}-$ deterministic $H$ )

\begin{tabular}{ccccccc}
\hline \hline$\Omega_{f_{\text {cul }}} \Omega_{I_{c}}$ & 0 & 0.1 & 0.2 & 0.3 & 0.4 & 0.5 \\
\hline 0 & 0.0 & 10.0 & 20.0 & 30.1 & 40.3 & 49.8 \\
0.1 & 5.0 & 15.1 & 20.6 & 30.4 & 40.6 & 50.0 \\
0.2 & 9.9 & 14.1 & 30.4 & 31.9 & 41.2 & 51.1 \\
0.3 & 14.9 & 17.8 & 25.1 & 36.3 & 46.5 & 53.1 \\
0.4 & 19.4 & 21.9 & 27.8 & 36.4 & 54.2 & 64.5 \\
0.5 & 23.9 & 26.0 & 31.5 & 39.5 & 57.7 & 81.2 \\
\hline
\end{tabular}

the random variables $I_{c}$ and $f_{c u}$ collectively increases $\Omega_{K}$ especially at large uncertainties in the relevant input random variables. This is dissimilar to the effect of such correlation assumption on $\Omega_{T}$ when estimated based on mutually relative errors in $M$ and $K$.

$\Omega_{K}$ is again estimated but applying MC simulation technique instead of the FOSM method and results for the un-correlated and perfectly correlated scenarios are given in Tables 2 and 3 . Comparing such results associated with a few selected discrete $\Omega_{f_{c u}}$ and $\Omega_{I_{c}}$ values to results shown in Figs. 3 and 4, it may observed that the FOSM method performs fairly good in capturing the relative error, $\Omega_{K}$, in the stiffness random parameter. Furthermore, results using either MC or FOSM techniques ascertain the fact that the contribution of the variation in $I_{c}$ to the variation in $K$ is much more pronounced than the contribution of the variation in $f_{c u}$. Furthermore, based on the set of 
retrieved values from the literature for $\Omega_{I_{c}}(=0.35)$ and $\Omega_{f_{c u}}(=0.18), \Omega_{K}$ assumes a value of 0.37 for the un-correlated hypothesis when adopting the FOSM method. This estimate for $\Omega_{K}$ is almost literally identical to that recovered when applying the MC simulation technique as shown in Table 2 (= 0.36). Both are even matching the literature estimate for $\Omega_{K}$ mentioned in Section 7 .

In a further informative step, Fig. 5 compares the resulting relative error, $\Omega_{K}$, in the stiffness parameter calculated using the FOSM method for un-correlated random variables $I_{c}, f_{c u}$, and $H$ for the two distinct cases of (1) deterministic (error-free) $H$ versus (2) random (uncertain) $H$ with a selected $\Omega_{H}=0.10$. Such selected value for $\Omega_{H}$, despite being larger than the value of 0.04 compiled from the literature and previously given in Section 7, is implemented herein in order to delineate an upper bound to $\Omega_{K}$ approving anticipated built-in modeling uncertainties in the height, $H$, of the frame under investigation. Results shown in Fig. 5 reveal a remarkable increase in $\Omega_{K}$ due to only $10 \%$ relative error (or variation) in $H$. An increase in $\Omega_{K}$ is still observed due to randomness in $H$ if adopting $\mathrm{MC}$ simulation technique, though such increase is fairly less than that detected in the case when FOSM method is applied (refer to Table 4). For instance, if relying on FOSM results, a noticeable jump in $\Omega_{K}$ from 0.36 (gathered from the literature) to 0.96 occurs for the case of uncorrelated random variables with the following combination of relative errors, namely: $\Omega_{I_{c}}=0.35$, $\Omega_{f_{c u}}=0.18$ along with assumed $\Omega_{H}=0.10$. This sharp rise in $\Omega_{K}$, along with $\Omega_{M}=0.1$, will directly negatively produce about $100 \%$ increase in $\Omega_{T}$ (going up from 0.18 to 0.36 ). On the other hand, if MC simulation is adopted, and again for the same relative errors mentioned above compiled from the literature, $\Omega_{K}$ will assume a value of about 0.48 (much less than the value predicted using

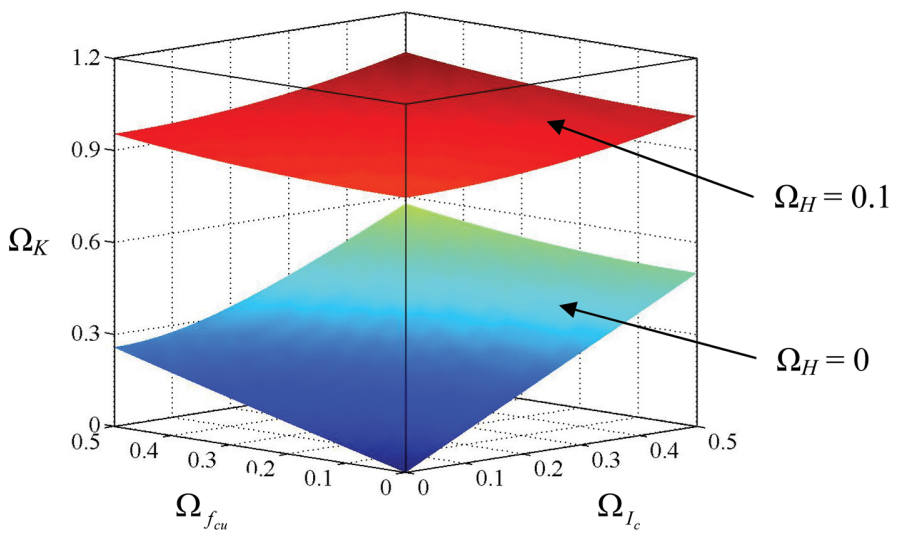

Fig. 5 Relative error in $K$ - Case of un-correlated $I_{c}, f_{c u}$ and $H$ with $\Omega_{H}=0$ and 0.10

Table 4 Values of $\Omega_{K} \times 100$ from MC simulation (un-correlated $f_{c u}, I_{c}$ and $H-\Omega_{H}=0.1$ )

\begin{tabular}{ccccccc}
\hline \hline$\Omega_{f_{\text {cu }}} \Omega_{I_{\text {c }}}$ & 0 & 0.1 & 0.2 & 0.3 & 0.4 & 0.5 \\
\hline 0 & 0.0 & 32.1 & 36.6 & 43.2 & 51.2 & 60.5 \\
0.1 & 30.6 & 32.6 & 37.0 & 43.5 & 51.5 & 60.7 \\
0.2 & 31.8 & 33.6 & 38.3 & 44.4 & 52.2 & 61.6 \\
0.3 & 33.6 & 35.4 & 39.6 & 45.8 & 53.6 & 62.5 \\
0.4 & 36.2 & 38.0 & 41.7 & 48.6 & 55.7 & 64.5 \\
0.5 & 40.2 & 41.7 & 45.1 & 51.8 & 58.6 & 67.2 \\
\hline
\end{tabular}


FOSM method). Such increase in $\Omega_{K}$, being relatively moderate compared to the value of 0.36 retrieved from the literature and assumed applicable to new designs, will cause only about $30 \%$ increase in $\Omega_{T}$. This increase in the built-in relative error in $T$ may un-conservatively affect seismic equivalent lateral force estimated based on the calculated period of vibration, $T$. When, however, MC simulation is repeated but for the actual value of $\Omega_{H}$ of 0.04 complied from the literature along with other values of 0.35 and 0.18 for $\Omega_{I_{c}}$ and $\Omega_{f_{c u}}$, respectively, the relative error $\Omega_{K}$ marks just a small increase $\left(\Omega_{K}=0.40\right)$ over its value of 0.36 gathered from the literature. The associated $\Omega_{T}$ will accordingly almost remain unchanged assuming a value of 0.19 for the set: $\Omega_{M}=0.1$ and $\Omega_{K}=0.36$ compiled from the literature, and a similar value in the order of 0.20 for the stochastically computed set with $\Omega_{M}=0.1$ and $\Omega_{K}=0.40$.

\section{Conclusions}

A set of mathematical equations is developed utilizing the theory of error propagation and FirstOrder Second-Moment techniques and introduced to determine bounds on the variance in theoretically calculated fundamental period of vibration for skeletal structures. Verifications and/or adjustments of these bounds are also carried out relying on the results recovered from applying the more precise Monte Carlo simulation technique. Studies of parametric uncertainties applied to investigated reinforced concrete frame bents are performed through both the FOSM and MC simulation approaches demonstrating the effect of randomness of various relevant structural and material properties on the relative error in the estimated period of vibration. Correlation between mass and stiffness - the two principal uncertain parameters of interest in the calculation of $T-$ is thoroughly discussed.

Generally speaking, it was found that the contribution of $\Omega_{M}$ and $\Omega_{K}$ to the relative error in the period, $T$, is theoretically similar if applying the FOSM method. Such conclusion has been also confirmed on a practical basis when applying the more precise MC simulation technique. Furthermore, it was found that estimated $\Omega_{T}$ values through either the FOSM approach or MC simulation technique assuming Lognormal PDF for input random variables are fairly comparable and almost identical despite the large uncertainty in these relevant input random variables considered in the present study. Moreover, referring to estimates of $\Omega_{M}$ and $\Omega_{K}$ available in the literature and from a practical perspective, the intrinsic uncertainty in the mass random parameter is comparably less than that in the stiffness parameter. Therefore, the effect of the relative error in $K$ on the resulting error (i.e. uncertainty) in the computed period of vibration is both theoretically and practically more pronounced than the contribution of the relative error in $M$. As a conclusion, and based on numerical information from the literature and the comparative error propagation analysis technique introduced in this paper, the relative error, $\Omega_{T}$, in the "theoretically" calculated period of vibration is in the range of 19 to $25 \%$ for the most likely hypothesis of "un-correlated" mass and stiffness parameters.

Furthermore, some random variables, namely: the frame height $H$; the moment of inertia, $I_{c}$, of the columns of the frame; and the compressive strength of concrete, $f_{c u}$, that directly affect the value of the stiffness parameter, $K$, have been investigated herein for the studied case of reinforced concrete frames. It is found that the resulting coefficient of variation, $\Omega_{K}$, is theoretically more sensitive to the relative error, $\Omega_{H}$, than to the relative error, $\Omega_{I_{c}}$; while the variation, $\Omega_{f_{c u}}$, has the least effect on the relative error in the estimated stiffness parameter, $K$. Such conclusion, found to 
be very comparable either if applying FOSM technique or MC simulation approach, is supported by numerical investigation with three-dimensional graphical and tabular representation of the results. Fortunately, practical level of inherent uncertainty in $H$ is numerically the least - as shown in the literature - which alleviates a bit the final total relative error in $K$ and henceforth in $T$. However, based on likely relative errors from the literature, $\Omega_{K}$ was found to reach a value of about 0.4 (refer to MC simulation results) and consequently $\Omega_{T}$ scores a value of 0.20 for the case of new designs. This may pose a risk on the safety and/or economy of designed facilities if such built-in uncertainty in the fundamental period of vibration, $T$, is un-conservatively overlooked, since seismic design base shear is generally based on an estimate of $T$. The base shear is very sensitive to any variation in $T$ if the point lies on the descending branch of the applicable seismic design response spectrum curve. Such conclusion applies to new seismic-resistant designs as well as to seismic risk assessment of existing structures, though the applicable value of $\Omega_{K}$ is different as presented in the body of the paper.

The uncertainty in seismic analysis/design due to inaccurate period estimation for a given skeletal structure is therefore an important subject worth to be investigated. For example, for new designs with a possible value of the relative error in the period of vibration of about 0.20 , and assuming that the period falls within the velocity-controlled region along with the assumption that the design spectrum decays inversely proportional to $T$, the difference in base shear values would be in the order of approximately $25 \%$. Such difference in base shear should nonetheless be evaluated versus the additional effects of the inherent variability, uncertainty and conservatism present in many other aspects of the codified seismic design process. This is currently under scrutiny by the author in an ongoing study aiming at quantifying the effect on the final design of the built-in error in the theoretically calculated period of vibration used to calculate seismic design loads (and hence used to size the structure) relative to the effects (i.e. contributions) of other inherently varying aspects typically encountered in (and mostly influencing) the seismic design process. These other aspects include: (1) intrinsic randomness in seismic design hazard and code specified importance factors for a particular structure; (2) code pre-specified built-in overstrength for new designs; (3) limits and bounds imposed by some codes on the estimated period of vibration such as in the US practice; and (4) code preset lower and upper bounds on design acceleration and drift, respectively, such as in Eurocode 8. The contributions of such variability and uncertainties should be nevertheless weighed against conservatism usually implemented in seismic design provisions. We would like however to highlight the fact that the present manuscript just focuses on the part dealing with quantifying the relative error (i.e. uncertainty) in theoretically calculated period of vibration, and hence determining and reporting bounds on the variance of such random variable through a comprehensive parametric study, for single mode-dominant skeletal structures due to inherent randomness in its constituents. Quantification of this error should be useful within the context of probabilistic approaches promoted in emerging seismic design provisions worldwide.

To conclude, this research is not only highlighting the presence of built-in uncertainties in theoretically calculated period of vibration, but is also quantifying bounds on these uncertainties (or variances) in such structural random property for single mode-dominant structures, and is further promoting to include such information about relative errors in $T$ in seismic design and rehabilitation standards for the emerging generation of performance-based codes already accepting and endorsing probabilistic approaches. Implementation of the paper's conclusion in relevant codes may be through explicitly listing bounds on expected relative errors in computed periods of vibration in order to quantify the most adverse effect on the seismic equivalent base shear due to such variance. 
The paper further offers specific estimates for such variability for a certain type of structural systems representing a large percentage of civil structures: single mode-dominant reinforced concrete skeletal structures such as those typically encountered in bridges, industrial facilities, elevated tanks, single-story dwellings, etc... Extensive computational and experimental efforts, accompanied by statistical interpretation both at the component as well as at the entire structure level, are still needed before establishing definite bounds on the relative error in the period of vibration for different structural systems including MDOF structures.

\section{Acknowledgements}

The author would like to acknowledge the fruitful e-mail discussions with Prof. G.G. Deierlein and the late Prof. C.A. Cornell of Stanford University at the initiation of this research especially regarding the section in the manuscript related to correlation between mass and stiffness.

\section{References}

ACI 318 (2005), "Building code requirements for structural concrete (318-05) and commentary (318R-05)", American Concrete Institute, Farmington Hills, MI, USA.

Adhikari, S. and Friswell, M. (2007), "Random matrix eigenvalue problems in structural dynamics", Int. J. Numer. Meth. Eng., 69(30), 562-591.

Ang, A.H. and Tang, W.H. (1984), "Probability concepts in engineering planning and design", John Wiley, New York, NY, Vols. I and II.

ASCE 7-05 (2006), "Minimum design loads for buildings and other structures", American Society of Civil Engineers, Reston, Virginia, USA.

Benjamin, J.R. and Cornell, C.A. (1970), "Probability, statistics, and decision for civil engineers", McGraw-Hill, Inc., USA.

CEN 2004 (2004), "European standard EN 1998-1: eurocode 8: design of structures for earthquake resistance. Part 1: General rules, seismic actions and rules for buildings", Comité European de Normalisation, Brussels.

Ellingwood, B., Galambos, T.V., MacGregor, J.G. and Cornell, C.A. (1980), "Development of a probability-based load criterion for American national standard A58", National Bureau of Standards, Washington DC, USA, 222.

Fardis, M.N. and Biskinis, D.E. (2003), "Deformation capacity of RC members as controlled by flexure or shear", Otani Symposium, 511-530.

Ghosh, D, Ghanem, R. and Red-Horse, J. (2005), "Analysis of eigenvalues and modal interaction of stochastic systems", AIAA J., 43(10), 2196-2201.

Goel, R.K. and Chopra, A.K. (1997), "Period formulas for moment-resisting frame buildings", J. Struct. Eng $A S C E, \mathbf{1 2 3}(11)$.

Haselton, C.B. and Deierlein, G.G. (2005), "Benchmarking seismic performance of reinforced concrete frame buildings", Proceedings of ASCE-SEI Structures Congress, NY, USA, April 2005.

Ibarra, L.F. (2003), "Global collapse of frame structures under seismic excitations", Ph.D. Thesis, Dept. of Civil and Envir. Engrg., Stanford University, CA, USA.

IBC 2003 (2004), "International building code", International Code Council, Falls Church, Virginia, USA.

Kareem, A. (1988), "Aerodynamic response of structures with parametric uncertainties," Structural Safety, 5, 205-225, Elsevier Science Pub., B.V., Amsterdam.

Kwon, O.S. and Kim, E.S. (2010), "Evaluation of building period formulas for seismic design", Earthq. Eng. Struct. Dyn., 39(14), 1569-1583.

Masi, A. and Vona, M. (2010), "Experimental and numerical evaluation of the fundamental period of undamaged 
and damaged RC framed buildings", B. Earthq. Eng., 8(3), 643-656.

Mirza, S.A., Hatzinikolas, M. and MacGregor, J.G. (1979), "Statistical description of strength of concrete", J. Struct. Div - ASCE, 105(ST6), 1021-1037.

Panagiotakos, T.B. and Fardis, M.N. (2001), "Deformations of reinforced concrete at yielding and ultimate", $A C I$ Struct. J., 98(2), 135-148.

Pinho, R. and Crowley, H. (2009), "Revisiting eurocode 8 formulae for periods of vibration and their employment in linear seismic analysis", E. Consenza (ed), Eurocode 8 Perspectives from the Italian Standpoint Workshop, 95-108, Doppiavoce, Napoli, Italy.

Shaikhutdinov, R. (2004), "Structural damage evaluation: theory and applications to earthquake engineering", Ph.D. Thesis, California Institute of Technology, Pasadena, CA, USA.

Soize, C. (2000), "A non-parametric model of random uncertainties for reduced matrix models in structural dynamics", Probab. Eng. Mech., 15(3), 277-294.

Tarantola, A. (2005), "Inverse problem theory and methods for model parameter estimation", SIAM.

UBC (1997), "Uniform building code", International Conference on Building Officials, Whittier, CA, USA.

$J L$ 